\title{
The effect of TiO2 and MgO on the thermoluminescence properties of a lithium potassium borate glass system
}

\begin{abstract}
The influence of dopant $\mathrm{TiO} 2$ and co-dopant $\mathrm{MgO}$ on the thermoluminescence (TL) properties of lithium potassium borate glass (LKB) is reported in this paper. The glow curve exhibits a prominent peak $(\mathrm{Tm})$ at $230^{\circ} \mathrm{C}$. The TL intensity was enhanced by a factor of $\sim 3$ due to the incorporation of $\mathrm{MgO}$, and this was attributed to the creation of extra electron traps mediated by radiative recombination energy transfer. We achieved good linearity of the TL yield with dose, low fading, excellent reproducibility and a promising effective atomic number (Zeff=8.89), all of which are highly suitable for dosimetry. The effect of heating rate, sunlight and dose rate on the TL are also examined. These attractive features demonstrate that our dosimeter is useful in medical radiation therapy.
\end{abstract}

Keyword: Semiconductors; X-ray diffraction; Luminescence 